\title{
Odeurs et représentations de l'Autre pendant la Première Guerre mondiale
}

\author{
Juliette Courmont \\ (Agrégée et titulaire d'un master en histoire au \\ Centre d'études historiques de l'EHESS)
}

\section{Résumé/Abstract}

[Fr] Pendant la Grande Guerre, la figure de l'animalité allemande rencontre un large succès dans les représentations françaises, car elle permet de mettre en système la plupart des reproches adressés à l'ennemi et de le retrancher de l'humanité. Une violence symbolique dans laquelle l'odeur, sens du contact, occupe une place particulière. Lors de l'invasion, l'odeur est perçue de manière indirecte comme une manifestation allemande, car les troupes laissent à dessein derrière elles des excréments dans les maisons envahies. Il s'agit là d'une pratique de cruauté d'une grande violence par ce qui est dit à l'Autre, ce qui est dit de l'Autre. Or l'interprétation des victimes est exactement opposée au message qui leur est adressé. Elles y lisent l'absence de contrôle de l'adversaire, un signe de son animalité. Par ailleurs, confrontés à la présence allemande dans les régions envahies ou au front, des Français dénoncent de façon spontanée une odeur intrinsèque de l'ennemi. Par sa dimension personnelle, la sensation olfactive permet d'approcher les modalités de perception de l'expérience de guerre. Présentant un caractère irréfutable, le discours olfactif contribue, lui, à la violence de la culture de guerre et nous permet d'en esquisser un peu plus les contours.

Mots-clefs : Première Guerre mondiale, odeur, altérité, ennemi, culture de guerre

[En] During the Great War, the figure of German animality is used to great effect in French portrayals, because it brings into play most of the critiques of the enemy and serves to cut him off from humanity. In this symbolic act of violence, smell, the sense of contact, plays a particular role. During the invasion, scent is indirectly viewed as a Germanic effect, because troops intentionally leave excrement behind them in the houses they invade. This is a cruel practice of great violence through what is said to and of the other. However, victims' interpretations are completely different from the message addressed to them through this act. Instead, they read here the absence of control of the adversary as a sign of his animality. Moreover, when directly confronted with the German, whether in invaded areas or at the front, the French spontaneously point out an intrinsic smell of the enemy. Through its personal dimension, olfactory feeling allows the bringing together of modes of percep- 
tion with the experience of war. The indisputable nature of olfactory speech contributes to the violence of the culture of war and allows us to outline it further.

Key words: First World War, smell/scent, culture of war, enemy, otherness/alterity

\section{Introduction}

«Nous nous débarrassons des mouches, mais nous ne pouvons éliminer une odeur fade très particulière : l'odeur de Boches. Je la reconnais bien pour l'avoir déjà sentie en 1917 lorsque je dormais sur la côte du Talou, dans une sape conquise aux Allemands. » ${ }^{1}$. «L'odeur de Boches », voilà ce que dénonce cet artilleur dans son carnet de guerre le 9 novembre 1918, à l'occasion de la reprise de Dercy dans l'Aisne. Une odeur singulière, à la fois identifiable et «fade », une odeur persistante, dérangeante. La remarque pourrait paraître anecdotique. Son auteur ne témoigne que d'une gêne, plus forte que celle des mouches certes, mais la sensation reste néanmoins passagère. Cependant en la regardant d'un peu plus près, on réalise que la mention dit plus que le désagrément ressenti par le combattant. De fait, elle établit une barrière anthropologique très efficace entre soi et l'adversaire. Le constat est dérangeant, et ce d'autant, que la remarque n'est pas isolée. Les chercheurs qui s'intéressent à la Première Guerre mondiale le savent bien, l'évocation d'une odeur des Allemands revient trop souvent sous la plume des contemporains de la période, pour qu'on puisse la mettre de côté comme on le ferait d'une aberration.

C'est pourtant sous cet aspect qu'elle est la plus visible aujourd'hui. En effet, théorisée dès 1915 par le médecin Edgar Bérillon, la réputation odorante des Allemands a fait l'objet de publications aux conclusions raciales radicales (La Bromidrose fétide de la race allemande, 1915 ; La Polychésie de la race allemande, 1915), qui ont rencontré un écho immédiat, mais également une relative postérité. La violence des thèses défendues, par exemple la nécessité de "l'organisation préventive et durable d'un certain nombre de mesures d'exclusion et d'élimination » pour la préservation de la race française (Bérillon, 1917, 63), et un sens certain de la formule, «l'Allemand urine par les pieds » (Bérillon, 1915, 11), ont d'abord assuré la publicité de ces écrits, avant d'en marginaliser leur auteur, réduit à l'image d'un excentrique, voire d'un savant fou. Une telle lecture ne résiste pas à une étude un tant soit peu approfondie, et les travaux du Docteur Bérillon constituent pour l'historien une source d'autant plus précieuse, que la recension des témoignages est assez ardue hors de la sphère médicale. Viser en ce domaine à l'exhaustivité est impossible, tant le champ d'expression est ouvert, des textes publiés aux documents personnels

\footnotetext{
${ }^{1}$ Fernand Laponce, Journal de marche d'un artilleur de campagne, Bois-Colombes, 1971, cité par Bruno Cabanes, «Ce que dit le contrôle postal », in Christophe Prochasson, Anne Rasmussen (dir), Vrai et faux pendant la Grande Guerre, Paris, La Découverte, 2004, note 38, p.70.
} 
(journaux, correspondances, dessins, objets...), mais il n'est toutefois pas si difficile de rassembler un corpus de documents suffisamment conséquent pour pouvoir esquisser les contours de la dénonciation de l'odeur des Allemands et tenter d'en comprendre le sens. Dans le cadre de notre réflexion consacrée dans un premier temps aux pratiques symboliques des violences de masse, nous privilégierons les sources qui émanent des combattants ou qui témoignent d'une confrontation avec ceux-ci.

L'examen de ces documents révèle que le reproche olfactif adressé par les Français aux Allemands participe de la construction d'une figure d'altérité hostile qui animalise l'adversaire. Un processus complexe où s'amalgament des accusations de natures différentes, que l'argument olfactif permet de relier. Avec l'odeur, les représentations de l'Allemand qui se sont élaborées progressivement depuis les débuts du $\mathrm{XIX}^{\mathrm{e}}$ siècle atteignent ainsi pendant la Première Guerre mondiale leur point d'orgue. Car, si on observe une permanence de la dénonciation olfactive dans le discours de mise à distance de l'Autre, déjà sensible à l'égard des Juifs au Moyen-âge, ou à l'égard des Noirs d'Amérique du Nord aux débuts du XX ${ }^{\mathrm{e}}$ siècle, c'est cependant la mise en système des accusations adressées à l'Autre, qui permet au processus de diabolisation de l'ennemi de franchir un palier. L'image ainsi construite peut d'ailleurs se prêter à des réemplois si les circonstances l'exigent, comme la figure de l'Allemand en 1914-1918 puise elle-même à des représentations plus anciennes. Certains discours tenus aujourd'hui dans les sociétés européennes à l'égard de l'étranger, qu'il soit lointain ou proche, témoignent ainsi que le rejet est possible : qu'on se souvienne par exemple de la désormais célèbre remarque de Jacques Chirac sur «le bruit et l'odeur» en 1991, ou qu'on écoute comme l'ethnologue Christian Bromberger les supporters italiens dans les stades : «Tu sens comme ça pue, [...] les Napolitains arrivent » $(2001,293)$. L'odeur n'est décidément pas un argument comme les autres.

Pour tenter d'en identifier les enjeux, nous observerons d'abord comment s'élabore la figure hostile de l'ennemi, en accordant une attention particulière à la part qu'y prend la violence. Nous nous attacherons ensuite à comprendre ce que les Français disent de l'Autre, ce qu'ils disent à l'Autre, quand ils dénoncent son odeur. Enfin, nous examinerons les caractères de cette odeur, et le symbolisme qui lui est intimement associé.

\section{Aux origines de la violence}

Dès les premières semaines de l'invasion en 1914, les exactions commises par l'armée allemande, d'abord en Belgique, puis dans le nord et l'est de la France établissent la brutalité de l'adversaire. Une violence, en quelque sorte immédiate, qui s'est d'abord traduite par le déploiement de pratiques extrêmes sur les lieux de combat à l'encontre des soldats blessés et/ou prisonniers. Émanant d'ordres supérieurs ou «spontanées », ces dernières consistent notamment en des mises à mort com- 
mises «à chaud» sur des soldats hors de combat, mais également en des meurtres commis «à froid », c'est à dire après le combat, par des soldats ou des officiers opérant en groupe ou isolément et cherchant à achever leurs adversaires immobilisés sur le champ de bataille. Ceux-ci sont alors exécutés par arme à feu, par arme blanche, par coups de crosse, comme en témoigne un nombre important de dépositions publiées par le troisième rapport de la commission d'enquête française daté du 1er mai $1915^{2}$. Des démonstrations de violence qui s'accompagnent aussi d'exécutions de soldats groupés, commises parallèlement à des massacres de civils. Une dimension collective qui montre que les prisonniers mis à mort ont été dans l'instant considérés comme collectivement complices d'une résistance perçue par les Allemands comme illégitime. Enfin, pour ce qui est des civils, le travail des historiens Alan Kramer et John Horne a permis d'estimer à 6000 environ le nombre de personnes massacrées, les victimes incluant dans certains cas des femmes et des enfants. À ces massacres, il faut ajouter les viols de masse, ainsi que les destructions de biens, d'incendies de maisons, de villages, ou de quartiers entiers. Les «atrocités allemandes », ainsi que les ont nommées les Alliés, fondent alors le discours français sur la sauvagerie de l'ennemi. Une sauvagerie extrême qui corrobore l'animalité de l'adversaire en le plaçant du côté des puants, c'est-à-dire selon l'ethnologue Bertrand Hell des carnivores, des nécrophages. Une comparaison qui n'est pas sans évoquer la consommation importante de viandes outre-Rhin et notamment de charcuterie. D'une certaine façon, «tout se passe comme si l'ordre social pouvait être le reflet d'un ordre pensé comme naturel » (Hell, 1994, 79).

Au même moment, d'autres pratiques de cruauté achèvent d'assimiler les Allemands à la pestilence. En effet, comme cela avait déjà été le cas en 1870-1871, les troupes laissent à dessein derrière elles des excréments dans les maisons des régions envahies. Des témoins interrogés par les magistrats de la très sérieuse commission d'enquête instituée fin septembre 1914 «en vue de constater les actes commis par l'ennemi en violation du droit des gens » détaillent alors la souillure de leur demeure par les contingents ennemis. Après avoir fait état de la destruction complète de son village par les soldats du 368ème régiment d'infanterie avant l'évacuation, le maire de Sempigny dans l'Oise termine ainsi son témoignage : "Les maisons ont été laissées dans un état de saleté répugnant. Il y avait des ordures partout, des excréments dans les tiroirs des meubles, dans les armoires et jusque dans notre boîte à lait. » ${ }^{3} \mathrm{La}$ violation du domicile et de l'intime, à travers les meubles, le linge, la chambre

\footnotetext{
${ }^{2}$ Rapports et procès-verbaux d'enquête de la commission instituée en vue de constater les actes commis par l'ennemi en violation du droit des gens, III-IV, Paris, Imprimerie nationale, 1916, pp.66-160.

${ }^{3}$ Rapports et Procès-verbaux d'enquête de la commission instituée en vue de constater les actes commis par l'ennemi en violation du droit des gens, VI-VII-VIII-IX, Paris, Imprimerie nationale, $1917, \mathrm{n}^{\circ} 96, \mathrm{p} .115$.
} 
même souvent, est durement ressentie par les Français qui en sont victimes. Et alors même que les déjections ont été laissées dans des lieux soigneusement choisis pour porter atteinte à l'intégrité de l'adversaire, ces pratiques donnent lieu à une interprétation inverse : les Allemands n'ont pas la maitrise de leurs sphincters. Une absence de contrôle de soi qui signerait leur bestialité. Le déni de l'agression volontaire est évident, et d'ailleurs arrangeant puisqu'il permet de compléter l'édification de l'animalité de l'ennemi. Une animalité malodorante en l'occurrence qui se combine avec la dénonciation d'une complaisance excrémentielle attestée, outre cette pratique de cruauté, par le pain KK qui alimente l'armée allemande à partir de 1915. Des initiales qui prêtent le flanc à de nombreux jeux de mots et d'images et qui contribuent à diffuser l'idée d'une proximité des Allemands avec l'excrément, voire même parfois d'une identité. L'ennemi est décidément bien un puant.

Les violences commises par l'ennemi à l'entrée en guerre ne suffisent cependant pas à expliquer comment la représentation des plus hostiles qui circule alors a pu s'élaborer aussi rapidement. Elle puise en effet ses racines à plusieurs sources. D'une part, la Première Guerre mondiale entretient une certaine proximité, et pas seulement temporelle avec la guerre de 1870-1871. On rencontrait déjà alors un certain nombre des reproches adressés à l'ennemi, sa bestialité, sa saleté, voire même son odeur comme on peut le lire par exemple sous la plume de l'historien Ernest Lavisse : "Sous sa tunique [...] l'Allemand garde sa chemise noire, grasse de sueur et de poussière, nauséabonde: aussi son odeur survit-elle longtemps à son départ » (1872, ch. XVII). Des souvenirs ravivés par l'entrée en guerre et l'invasion de 1914 et qui prennent désormais un sens nouveau comme l'illustre ce témoignage d'une transparence rare d'Isabelle Rimbaud en 1916 :

À Reims. Sur la place du parvis, à côté de la statue de Jeanne d'Arc, de nombreux soldats ennemis sont campés, leurs fusils en faisceaux alignés près d'eux. De l'ensemble du campement se dégage une odeur particulière, désagréable, écœurante. Elle me rappelle les relents détestés en 1870, époque à laquelle toute petite, je ne savais où me cacher pour échapper à la puanteur des Allemands logés chez nous à Charleville. Nous attribuions alors cette odeur à leur nourriture assez misérable, comme l'était d'ailleurs leur équipement. Aujourd'hui qu'ils marquent l'opulence et observent l'hygiène la plus stricte, les relents sont les mêmes et aussi accentués ${ }^{4}$.

Par ailleurs, la figure de l'Allemand qui circule dans la société française pendant la Grande Guerre emprunte aussi aux représentations qui ont cours pendant l'affaire Dreyfus où tout un corpus d'éléments permet de mettre à distance les «dreyfusards»: animalité et complaisance avec l'excrément, notamment. Plus que l'adversaire du moment, l'Allemand incarne donc l'ennemi dans son universalité. Et

\footnotetext{
${ }^{4}$ Isabelle Rimbaud, « Dans les Remous de la bataille », in Mercure de France, 16 août 1916, cité par La Chronique médicale, $1^{\text {er }}$ octobre 1916.
} 
la violence des représentations qui lui sont associées, au travers notamment d'une surprésence du discours olfactif, dépasse alors la seule réponse aux violences subies dans la guerre.

\section{Diaboliser l'Autre, une pratique de guerre}

En suspendant les normes établies en temps de paix, la guerre amène une redéfinition des règles qui peut être lue comme une forme de régression, ainsi que le suggérait Marc Bloch. Et l'on pourrait reprendre au bénéfice du préjugé olfactif sa formule : «La fausse nouvelle est le miroir où la «mémoire collective » contemple ses propres traits $\gg$ (Bloch, 1999 [1921], 56). En effet, la perception d'une pestilence de l'ennemi et sa circulation reflètent avant toute chose la peur de l'Autre. La vulnérabilité accrue des populations engagées dans la guerre les portant à accroître leur méfiance vis à vis d'autrui, elles sont plus facilement enclines à créditer l'existence d'une altérité olfactive. Or, si une odeur dit quelque chose à celui qui la respire, le discours olfactif est tout autant révélateur. Ainsi, à travers la dénonciation de la puanteur allemande, c'est non seulement la sauvagerie de l'adversaire, mais aussi leur propre peur que les populations françaises expriment. Une peur porteuse de haine. Affirmer que l'ennemi pue, c'est le rejeter au plus loin de soi et légitimer l'expression d'une violence verbale et physique sans limite.

L'historien allemand Michaël Jeismann a établi que pour les Français, l'ennemi est perçu comme un barbare depuis les guerres révolutionnaires, et nous avons vu avec quel profit la Première Guerre mondiale avait réinvesti ce topos. Le terme employé comme un négatif de l'image que les Français ont d'eux-mêmes exclut inévitablement l'Autre de l'humanité. Il le définit comme un Nicht-Mensch, celui qui ne peut être un homme. Et dans cette dialectique du conflit désormais pensée en termes anthropologiques, l'argument olfactif occupe une place de choix. Car stigmatiser l'odeur des Allemands, c'est toucher à des réflexes parmi les plus ancrés de la perception de l'altérité. Une coupure eux/nous, indispensable semble-t-il à l'activité guerrière qui se retrouve de manière particulièrement nette sous la plume de Françoise Héritier, lorsque celle-ci s'interroge sur «les matrices de l'intolérance et de la violence » :

On sait en effet que le propre de toute société humaine quelle que soit sa taille est de réduire la définition de l'humain aux membres du groupe, les autres étant des non humains qu'il est possible de traiter comme tels. Le terme utilisé par une ethnie pour se désigner signifie généralement tout simplement «les hommes ». [...] Chaque groupe humain est ainsi investi, croit-il, de l'humanité à l'exclusion de tout autre (Héritier, 1999, 330).

En historien, on ne peut que constater à quel point cette matrice agit dans le cadre de la guerre moderne, et de la Première Guerre mondiale tout particulièrement. Elle explique les franchissements des seuils de violence induits par cette aptitude à ce re- 
tranchement de l'humanité de ceux qui ne sont pas «des hommes». Un retranchement que l'odeur, à sa manière, opère à la perfection.

Or dénoncer la puanteur de l'ennemi, c'est non seulement lui adresser une parole de haine, mais souvent aussi la promesse d'une violence à subir. On le lit sans détours dans cette lettre du 4 avril 1918, classée par le contrôle postal dans la rubrique «bon moral» :

J'ai les idées belliqueuses, je voudrais bouffer du Boche, je voudrais voir leur sale gueule, il paraît qu'ils s'en font mettre, salauds, cochons, qu'est-ce que je leur passerais, je plains ceux qui auraient le malheur de tomber entre mes mains. Ah ! Si jamais nous allons en Bochie nous allons leur faire voir un peu à ces salauds de quel bois on se chauffe, ils ont violé nos filles, déshonoré nos femmes, nous en ferons autant ; pour ma part je fais venir un stock de capotes car j'aurais trop peur de me salir à leur contact de ces superbes gretchens, il paraît qu'elles ont du rabiot de gras double et qu'elles sentent toutes l'aigre $(\ldots)^{5}$.

Mais, que penser de ce soldat qui demande à sa femme de montrer «à notre cher petit Martin », la photographie au recto de la carte postale d'une fillette juchée sur un casque à pointe comme sur un pot de chambre et invitant Guillaume à goûter de ce pot de confiture $?^{6}$ S'agit-il de légitimer la violence que ce père a pu être amené à infliger à l'ennemi ? De transmettre le mépris dans lequel on tient l'adversaire ? La culture de guerre qui s'élabore pendant la Première Guerre mondiale abonde de ce type d'exemple. Ils disent pour le moins l'engagement de la société dans son ensemble au service du conflit (Audoin-Rouzeau \& Becker, 1997).

\section{Une odeur intolérable}

La récurrence de la dénonciation d'une puanteur allemande pendant la Grande Guerre pose avec acuité la question de sa réalité. En effet, même en se limitant aux propos de ceux qui disent en avoir fait l'expérience, les combattants et les occupés principalement, le discours semble parfois empreint d'une influence extérieure. C'est le cas dans le témoignage de Marie Masquelier, une habitante de Lille, qui note dans son journal, le 28 mars 1915 : «Une odeur peu agréable flottait dans l'air. C'est ainsi partout où il y a des Allemands je crois » ${ }^{7}$. La formulation renvoie à un savoir dont l'origine est mal connue, mais qui ne peut manquer d'avoir influencé la perception, ou tout au moins, la lecture qui en a été faite. Il en va de même pour Mademoiselle Prudhommeaux, institutrice à Guise dans l'Aisne, selon laquelle

\footnotetext{
${ }^{5}$ SHAT, $16 \mathrm{~N} 1395$, II ${ }^{\text {ème }}$ Armée, $224{ }^{\text {ème }}$ Rgt d'Artillerie.

${ }^{6}$ Collection particulière Bruno de Perthuis.

${ }^{7}$ Journal de Marie Masquelier cité par David Bellamy (2010), «Marie Masquelier, une jeune femme à Lille dans la Grande Guerre », dans Trévisi, M., Nivet, P. (dirs.), Les femmes et la guerre de l'Antiquité à 1918, Paris :, Economica, pp.325-344.
} 
«partout règne une odeur malpropre de sueur et de tabac, l'odeur boche pénétrante, qui résiste encore ${ }^{8}$. Le terme d' " odeur boche » indique une connaissance externe de la sensation et l'on peut en déduire, comme Philippe Nivet que «ce témoignage est révélateur de l'image de l'ennemi véhiculée par la culture de guerre » (Nivet, $2005,89)$. Une thèse corroborée par la lettre d'un artilleur précédemment citée où l'auteur employait le terme «il paraît » au sujet des caractères attribués aux femmes allemandes. Toutefois, la question du substrat sur lequel se projettent ces représentations reste en suspens. Rien ne permet en effet d'établir que la puanteur dénoncée soit inventée, ni qu'elle soit objective.

Dans une situation analogue d'invasion, Henriette Thiesset, âgée de 12 ans, écrit dans son journal après que les soldats qui occupaient sa maison fussent partis : «Ce départ fut pour nous un véritable soulagement, mais il restait la chambre à nettoyer et l'odeur d'Allemand y persista plus d'une semaine avec la fenêtre ouverte » ${ }^{9}$. Manon Pignot, qui a étudié ce journal, souligne que tous les topoï de la barbarie allemande y sont évoqués. Néanmoins, l'indication de durée portée par l'adolescente plaide pour une sensation véritable. Interrogée par l'historienne en 2004 sur son évacuation de 1918, une autre enfant de la guerre, Antoinette B. évoque spontanément l'odeur allemande. Questionnée sur sa réalité, elle décourage la lecture métaphorique que l'on serait tenté de faire aujourd'hui.

— «Donc, ce n'était pas une métaphore?

— Non, c'était vraiment une odeur qui imprégnait tout. [...]

— Je l'ai lu ailleurs mais je pensais que c'était une métaphore...

- Ah non, c'est vrai, ils avaient une odeur très forte, très forte, qui imprégnait tout.

C'était peut-être le harnachement de cuir, peut-être qu'ils fumaient un tabac spécial, ils étaient grands buveurs de bière aussi... ${ }^{10}$

Chez les combattants, les témoignages insistent eux aussi, sur la persistance et l'intensité de l'odeur de l'ennemi. Un poilu écrit depuis le front le 14 juillet 1915 : « J'ai voyagé en Bochie et j'ai été à même de constater l'odeur nauséabonde dégagée par ses habitants. Cette odeur, pour moi, est la même que celle du porc. [...] À tel point qu'on ne peut se rendre compte, si ce sont les Boches qui sont des cochons ou si ce sont les cochons qui sont des Boches ${ }^{11}$. Une virulence que l'on retrouve chez

\footnotetext{
${ }^{8}$ Témoignage de $\mathrm{M}^{\text {elle }}$ Prudhommeaux, institutrice, cité par O. Jonneaux, l'occupation allemande à Guise, maîtrise sous la direction de S. Audoin-Rouzeau et A. Duménil, Université de Picardie, 2000, p.93, repris par Philippe Nivet (2005).

${ }^{9}$ Journal. Bibliothèque de Documentation Internationale Contemporaine, côte F delta (en triangle) 1126/7C 695, cité par Manon Pignot, «Avoir 12 ans dans Ham occupé : le journal de guerre d'une jeune picarde, Henriette Thiesset (1914-1919) », in Carpi, O., Nivet, P. (dir), op. cit., pp.137-146.

${ }^{10}$ Antoinette B. extrait de l'entretien réalisé avec Antoinette et Jeanne B. nées en 1905 et 1918, par Manon Pignot le 11 avril 2004 à Vaucottes-sur-mer, p.76.

${ }^{11}$ Témoignage d'un poilu le 14 juillet 1915, cité par le Bulletin des Armées de la République, juillet 1915, et reproduit dans «l'odeur des Allemands », Revue de Psychothérapie, janvier 1923, p.14.
} 
le capitaine Augustin Cochin, avec toutefois un peu plus de précisions : "C'est ennuyeux de se faire tuer derrière le parapet par de tels animaux. Ils ont une odeur spéciale, très forte, dont on ne peut plus se défaire, quand on vit comme nous dans leurs lignes $»^{12}$. À lire ces témoignages, la thèse d'une perception olfactive véritable s'impose donc.

Pourtant, quant à définir cet effluve, les témoignages divergent. Cela peut s'expliquer par la difficulté à décrire les sensations olfactives, faute d'une taxinomie satisfaisante. Mais en étant attentif à ce que disent les témoins, on observe surtout que le caractère invasif et tenace de l'odeur allemande prime sur la senteur proprement dite. On peut le comprendre par la corporéité de l'expérience olfactive : l'odeur revêt une forte connotation identitaire pour les autres et, pour soi. Être imprégné de l'odeur de l'Autre, c'est déjà une atteinte majeure, presque vitale. Les contemporains de la Première Guerre mondiale ne sont d'ailleurs pas une exception en la matière. Les sociétés accordent en effet une place particulière aux odeurs du corps humain et de ses produits : excréments, urine, sueur... Elles sont toujours classées négativement et sont particulièrement bien mémorisées par ceux qui en font l'expérience. Les enquêtes menées à ce sujet auprès de professionnels par l'anthropologue Joël Candau sont éloquentes. Infirmières, médecins, sapeurs-pompiers, se disent unanimement marqués par certaines odeurs humaines, qu'il s'agisse de pathologies, de sang, de la pourriture de la chair ou d'odeurs de personnes étrangères aux règles d'hygiène communément en vigueur. Particulièrement nauséabondes, elles ont provoqué ou provoquent encore chez eux un malaise, des nausées, ou même une sorte d'ictus olfactif dans le cas de ce qui est identifié par l'expression d'«odeur de sale». Si les mots manquent, les sensations, elles, laissent une forte empreinte chez ceux qui les ont éprouvées. La dénonciation du caractère tenace de l'odeur allemande révèle donc à quel point elle constitue une agression pour les Français : elle viole le domicile, elle franchit la barrière du corps. Et comme l'empiètement olfactif est vécu sur le mode d'une sorte de menace, il est particulièrement bien mémorisé. Ce fut le cas notamment pour Fernand Laponce par lequel nous avons commencé cette réflexion. La catégorisation est alors forcée par le contexte, car l'odeur ne peut être détachée du substrat dont elle émane. Toute odeur nouvelle perçue dans le sillage de l'armée ennemie lors de l'invasion, de l'occupation ou du combat, a donc les plus grandes chances d'être identifiée comme «odeur de boche » et d'être codée négativement quelles que soient les exhalaisons en cause. L'impression olfactive devient ici emblématique de la situation globale et cristallise ses enjeux, à l'insu parfois des acteurs eux-mêmes. Dans ces conditions, la dénonciation de l'odeur de l'Allemand en 19141918 serait la projection de l'agression ressentie sur une odeur ordinaire. Elle sym-

${ }^{12}$ Le Capitaine Augustin Cochin, quelques lettres de guerre, s.l., Bloud et Gay, 1917. Lettres des 6 et 7 juillet 1916, envoyées du front de la Somme, cité par Stéphane Audoin-Rouzeau et Annette Becker, 14-18, retrouver la guerre, Paris, Gallimard, 2000, pp.199-200. 
boliserait l'agression de l'invasion, voire de l'occupation. Le témoignage d'Antoinette B. est significatif à cet égard. Evoquant le moment de l'évacuation en 1918, avec toute sa famille, après quatre années d'occupation, la délivrance est décrite sur le mode olfactif : «Oh ! Extraordinaire ! Et ma petite sœur qui avait 4 ans a dit - parce que les Allemands avaient une odeur très particulière, ils sentaient très mauvais - alors elle a dit : "ça ne sent plus le boche ici !". Ça l'a frappée, en arrivant en Suisse ${ }^{13}$.

\section{Conclusion}

Contrainte par le contexte, la dénonciation de «l'odeur de Boche » est à la mesure de l'agression ressentie lors du contact avec l'ennemi. Le caractère insupportable de cette odeur est en outre renforcé, voire orienté, dans un grand nombre de situations par l'ennemi qui s'identifie de lui-même comme puant à travers des pratiques de souillure des habitations violées. Faits sociaux en tant que tels, les odeurs subissent l'influence de la mobilisation idéologique et patriotique, en même temps qu'elles y contribuent. La dénonciation olfactive participe donc de la violence de la culture de guerre française, sans doute l'une des plus brutales que l'on puisse trouver parmi tous les belligérants. En effet, si la pratique de souillure excrémentielle est assez fréquemment attestée dans les conflits, l'essentialisation de l'ennemi à travers son odeur est quant à elle bien moins courante.

Une culture de guerre qui a littéralement « rendus brutaux »-selon la traduction en français du concept de «brutalisation » initié par George Mosse - ceux qui s'y sont intégrés et l'ont portée.

Mais au travers de l'odeur des Allemands, c'est aussi l'odeur d'un ennemi universel qui est en jeu. Certes, ici ou là, les odeurs de certains groupes humains avaient déjà été stigmatisées, mais en intégrant la dimension olfactive à un système de représentation complexe, la dénonciation prend une tout autre portée. Elle invite donc à s'interroger sur la place qu'occupe la puanteur dans le système de représentation du monde des Européens, et des Français notamment.

\section{Bibliographie}

\section{Sources primaires :}

BÉRILlON, E. (1917). La Psychologie de la race allemande d'après ses caractères objectifs et spécifiques, Paris : Maloine, p.63.

\footnotetext{
${ }^{13}$ Antoinette B., op.cit. Elle répond à la question : «Qu'avez-vous ressenti à ce moment-là ? ».
} 
BERILlON, E. (1915). La Bromidrose fétide de la race allemande, Paris : Maloine, p.11.

BLOCH, M. (1999 [1921]). «Réflexions d'un historien sur les fausses nouvelles de la guerre », Revue de synthèse historique, réédité par Allia, 56 p.

LAVISSE, E. (1872). L'Invasion dans le département de l'Aisne, Laon : H. Coquet.

\section{Sources secondaires :}

Audoin-RouzeAu, S., Becker, A. (1997). «Violence et consentement : culture de guerre du premier conflit mondial », in RIOUX, J-P., SiRINELlE, J-F., (dir.), Pour une histoire culturelle, Paris : Seuil, pp.251-271.

Audoin-RouzeAu, S., BECKER, A. (2000), 14-18 Retrouver la guerre, Paris : Gallimard.

BROMBERGER, C. (2001). Le match de football, ethnologie d'une passion partisane à Marseille, Naples et Turin, Paris : MSH, 293 p.

CANDAu, J. (2000). Mémoire et expériences olfactives, Anthropologie d'un savoir faire sensoriel, Paris : PUF.

HELL, B. (1994). Le sang noir : chasse et mythe du sauvage en Europe, Paris: Flammarion.

HÉRITIER, F. (1999), De la violence II, Paris : Odile Jacob.

Horne, J., Kramer, A. (2001). German Atrocities, 1914: A History of Denial, New Haven : Yale University Press.

JeISMann, M. (1997). La Patrie de l'ennemi, la notion d'ennemi national et la représentation de la nation en Allemagne et en France de 1792 à 1918, Paris : CNRS éd.

NiVET, P. (2005). «Vivre avec l'ennemi : les relations entre occupants et occupé en Picardie (1914-1918) », in CARPI, O., NIVET, P., (dir), La Picardie occupée : du Moyen-âge au XX siècle, actes du colloques d'Amiens du 13 juin 2003, Amiens : Encrage, pp.81-136. 